\title{
Effect of Lactobacillus rhamnosus on the response of Galleria mellonella against Staphylococcus aureus and Escherichia coli infections
}

\author{
Adeline Lacerda Jorjão ${ }^{1} \cdot$ Felipe Eduardo de Oliveira $^{1}$ - Mariella Vieira Pereira Leão ${ }^{2}$. Antonio Olavo Cardoso Jorge ${ }^{1}$. \\ Luciane Dias de Oliveira ${ }^{1}$
}

Received: 21 March 2017 / Revised: 9 September 2017 / Accepted: 19 October 2017 / Published online: 24 November 2017

(c) Springer-Verlag GmbH Germany 2017

\begin{abstract}
This study evaluated the prophylactic effects of the live or heat-killed probiotic strain Lactobacillus rhamnosus ATCC 7469 in Galleria mellonella, inoculated with Staphylococcus aureus or Escherichia coli. L. rhamnosus suspension was prepared and a part of it was autoclaved to obtain heat-killed lactobacilli. The larvae were inoculated of these suspensions and pathogenic. The survival of the larvae was observed during 7 days and after $24 \mathrm{~h}$ of inoculation haemocytes counted, melanization and nitric oxide production were analyzed. Larvae survival rate increased in the group inoculated with heat-killed L. rhamnosus, however, with no statistical difference. There was a significant increase in total haemocyte counts in all test groups. Haemolymph melanization and nitric oxide production were higher in the group inoculated with $L$. rhamnosus and infected with $S$. aureus. It was concluded that, in this model of infection, heat-killed L. rhamnosus ATCC 7469 promoted greater protection in Galleria mellonella infected with S. aureus or E. coli.
\end{abstract}

Keywords Lactobacillus rhamnosus $\cdot$ Galleria mellonella $\cdot$ Infection $\cdot$ Curve survival $\cdot$ Melanization

\section{Introduction}

Probiotics are living microorganisms that show beneficial effects on the host when administered in adequate quantities and they have attracted increasing interest in its beneficial effects (Nakayama et al. 2014; FAO; WHO 2001). Scientific evidences demonstrate that these microorganisms, even when inactivated, positively influence host health (Taverniti and Guglielmetti 2011; Dash et al. 2015). Probiotics have been used as a therapeutic alternative in inflammatory bowel diseases, and studies have shown that different probiotic bacterial strains have immunomodulatory effects (Harb et al. 2013; Dash et al. 2015; Jorjão et al. 2015). Adams (2010)

Communicated by Jorge Membrillo-Hernández.

Adeline Lacerda Jorjão

adelinejorjao@gmail.com

1 Department of Biosciences and Oral Diagnosis, Laboratory of Microbiology and Immunology, Institute of Science and Technology, Paulista State University/UNESP, Av, Engenheiro Francisco José Longo, 777-Jardim São Dimas, São José dos Campos, SP 12245-000, Brazil

2 Bioscience Basic Institute, University of Taubaté, Rua Expedicionário Ernesto Pereira, 3, Taubaté, São Paulo, Brazil suggests that live probiotic cells may influence the gastrointestinal microbiota and immune response, whereas the dead cell components have an anti-inflammatory response in the gastrointestinal tract. Thus, variable amounts of dead or living cells could generate different responses, and the use of inactivated probiotic bacteria is considered safer.

The use of insects as models of experimental infection has shown great growth in research involving human pathogens an it has became a valuable alternative compared to animal models, which are currently more expensive and involve ethical conflicts (Junqueira et al. 2012). Insects have a relatively advanced system of antimicrobial defenses because they have innate immune systems and hemolymph cells, which are able to encapsulate or phagocyte microbial invaders, as well as adaptive responses that include the induced production of lysozyme and small antimicrobial peptides (Ramarao et al. 2012). Few studies have used this model to study the effects of probiotic bacteria (Vilela et al. 2015; Köhler 2015).

Pathogenic microorganisms are very common and they are capable to colonize and infect tissues of the host and cause disease. There is increasing evidence that there is a mutual relationship between beneficial and pathogenic bacteria in the bowel of mammals and insects, which are 
controlled by the immune system. Deciphering these complex interactions between the intestinal microbiota and the immune system requires systems or models of infection, such as G. mellonella larvae (Köhler 2015).

Thus, it is of great interest to enlarge the studies with $G$. mellonella on the action of alive or heat-killed probiotics to verify their influence on the immune response against a bacterial challenge. This study evaluated the effects of the alive or heat-killed probiotic strain Lactobacillus rhamnosus ATCC 7469 on Galleria mellonella, by means of survival curve of these larvae when treated with $L$. rhamnosus and challenged with $S$. aureus or $E$. coli, bacteria widely used in research in our laboratories and of different groups, as well as the influence in the response to these microorganisms, analyzing the total count of haemocytes, production of nitric oxide and hemolymph melanization.

\section{Materials and methods}

\section{Galleria mellonella}

Galleria mellonella is a species of lepidopteran insects, more specifically of moths, belonging to the family Pyralidae, holometabolic, oviparous. It occurs naturally in hives of bees, feeding on old wax, pollen and honey, eggs come caterpillars, which, after a series of transformations, reach full development. The last stage of the G. mellonella larval is used as an experimental model for the virulence studies of microorganisms and antimicrobial potency. In our study, caterpillars were used in our Invertebrate Laboratory of the Department of Bociences and Oral Diagnosis, at the ICTUNESP. By means of an already standardized breeding protocol, when the caterpillars reach a weight of around $0.300 \mathrm{~g}$ they are used in the experiments.

\section{Lactobacillus rhamnosus (live and heat killed), S. aureus and $E$. coli suspensions}

A standard strain of $L$. rhamnosus ATCC 7469 was seeded in Man-Rogosa-Shape Agar (MRS-Oxoid, Basingstroke, Hampshire, England) and grown at $37{ }^{\circ} \mathrm{C}$ in $5 \% \mathrm{CO}_{2}$ for $24 \mathrm{~h}$. From the colonies isolated and confirmed by Gram staining, a suspension in sterile and pyrogenic PBS was standardized to the concentration of $10^{8}$ cells $/ \mathrm{mL}$ in a spectrophotometer with a wavelength of $540 \mathrm{~nm}$. To obtain the heat killed probiotic suspension, part $(10 \mathrm{~mL})$ of the above suspension was autoclaved at $121{ }^{\circ} \mathrm{C}$ for $15 \mathrm{~min}$ and then centrifuged for $10 \mathrm{~min}$ at $5000 \mathrm{rpm}$ and the pellet resuspended in sterile and pyrogenic PBS.

Standard strains were also used, kept in a freezer at $-70{ }^{\circ} \mathrm{C}$ in the Laboratory of Microbiology and Immunology of the São José dos Campos/UNESP Institute of
Science and Technology, S. aureus ATCC 6538 or E. coli ATCC 25,922 seeded on BHI agar (M211-HIMEDIA) and from colonies isolated and confirmed by gram staining were standardized in PBS at $10^{9}$ cells $/ \mathrm{mL}$ in spectrophotometer. Before finding this concentration, an analysis of the susceptibility of $G$. mellonella to these microorganisms was carried out, and then the sub-lethal concentration of both bacteria was determined.

\section{Survival curve}

A hundred and eight larvae were used in the final stage of larval phase with with body weight of around $0.280-0.320 \mathrm{~g}$, with 12 larvae per group. In the interaction between $L$. rhamnosus (alive or heat-killed) and pathogenic bacteria ( $S$. aureus or E. coli), $10 \mu \mathrm{L}$ of the standardized probiotic bacteria were inoculated with precision syringes (Hamilton Inc., USA) in the hemolymph of each larvae through the last left proleg. After $2.5 \mathrm{~h}, 10 \mu \mathrm{L}$ of standardized suspensions of $S$. aureus or E. coli were inoculated in the last right proleg. For the control groups $10 \mu \mathrm{L}$ of PBS were inoculated. A total of nine experimental groups were performed, as described in Table 1. The number of dead G. mellonella was first recorded $18 \mathrm{~h}$ after inoculation of the microorganisms and then every $24 \mathrm{~h}$ and during 7 days after the beginning of the experiment. The larvae were considered dead when there was no movement to the touch. The death of all larvae of the experimental group or the transition to the pupal form determined the end of the experiment.

Table 1 Distribution of experimental groups

\begin{tabular}{lll}
\hline $1^{\circ}$ inoculation & $\begin{array}{l}2^{\circ} \text { Inoculation (após } 2 \mathrm{~h} \mathrm{e} \\
\end{array}$ & \\
\hline $\begin{array}{l}\text { L. rhamnosus } \\
\text { Alive }\end{array}$ & & \\
& PBS & AL (control) \\
& S. aureus & ALS (test) \\
& E. coli & ALE (test) \\
Inactivated & PBS & HK (control) \\
& S. aureus & HKS (test) \\
& E. coli & HKE (test) \\
& & \\
PBS & S. aureus & S (control) \\
& E. coli & E (control) \\
& PBS & PBS (control)
\end{tabular}




\section{Collection of haemolymph, total haemocyte count, analysis of haemolymph melanization and production of nitric oxide}

Twenty-four hours after inoculation of the larvae, euthanasia $(n=12)$ was performed to collect hemolymph. The larvae were immobilized in refrigerated Petri dish for $20 \mathrm{~min}$, then, with a scalpel blade, incisions were made in the ventral part. The larvae were squeezed, the hemolymph was removed, transferred to refrigerated microtubes, centrifuged at $9500 \times \mathrm{g} / 10 \mathrm{~min}$ at $4{ }^{\circ} \mathrm{C}$ and used for further analyzes.

For total haemocyte count, the pellet resuspended in $1 \mathrm{~mL}$ of cooled IPS (sterile anticoagulant buffer: $150 \mathrm{mM}$ sodium chloride, $5 \mathrm{mM}$ potassium chloride, $10 \mathrm{mM}$ tris- $\mathrm{HCl}, \mathrm{pH}$ 6.9, $10 \mathrm{mM}$ EDTA And $30 \mathrm{mM}$ sodium citrate) was used. New centrifugation $\left(9500 \times g / 10 \mathrm{~min}\right.$ at $\left.4{ }^{\circ} \mathrm{C}\right)$ was performed and the pellet resuspended again in $1 \mathrm{~mL}$ of IPS and cell viability was assessed by exclusion test with tripan blue. Total haemocyte count was performed on a cell counter (Countess Cell-Invitrogen).

For the quantification of melanization, the supernatant diluted in IPS ( $10 \mu \mathrm{L}$ of hemolymph in $40 \mu \mathrm{L}$ of IPS) was added to 96 -well plates $(n=8)$. After $5 \mathrm{~min}$, the optical densities of the wells were read in spectrophotometer with a wavelength of $405 \mathrm{~nm}$.

The concentration of nitric oxide was determined using Griess test. An aliquot of $100 \mu \mathrm{L}$ of the hemolymph supernatant was diluted in $900 \mu \mathrm{L}$ of IPS and $100 \mu \mathrm{L}$ aliquots of the samples were placed in a 96-well microplate $(n=8)$. The same volume of Griess reagent was added and after $10 \mathrm{~min}$, the absorbance was measured on a microplate reader with wavelength of $570 \mathrm{~nm}$. Nitrite standard samples ranging from 100 to $0.781 \mu \mathrm{M}$, diluted in hemolymph of uninfected larvae, were used to calculate the nitrite concentration, forming a standard curve.

The survival curve and estimation of differences in survival were determined by Log-rank test (Mantel-Cox). The results of total haemocyte counts, hemolymph melanization analysis and nitric oxide production were statistically analyzed by ANOVA, complemented by Tukey test. The significance level adopted in all analyzes was 5\% $(p \leq 0.05)$. All statistics were performed with GraphPad Prism 5.0 software.

\section{Results}

The numbers of dead larvae during S. aureus and E. coli infection at different times and experimental groups and survival percentage obtained at the end of the experiment are shown in Table 2. Figure 1a, b demonstrate the survival curves of larvae, when inoculated with alive or heatkilled $L$. rhamnosus and only with pathogenic bacteria. There was no statistical difference among the groups, but
Table 2 Survival percentage of G. mellonella infected with $S$. aureus or E. coli, in the different experimental groups, with or without previous inoculation of alive or heat-killed $L$. rhamnosus

\begin{tabular}{ll}
\hline Group & Percentage (\%) \\
\hline PBS (control) & 100 \\
AL (control) & 91.67 \\
HK (control) & 87.5 \\
S (control) & 50 \\
E (control) & 58.33 \\
ALS (test) & 25 \\
ALE (test) & 50 \\
HKS (test) & 58.33 \\
HKE (test) & 75 \\
\hline
\end{tabular}

it can be observed that when previously inoculated with the inactivated lactobacilli, there was an increase in the survival rate of larvae.

In the groups inoculated with alive lactobacilli and challenged by $S$. aureus (ALS), larvae mortality was statistically higher $(p \leq 0.05)$ than the group inoculated only with $S$. aureus (Sa). The reduction in mortality was also observed in the group inoculated first with alive lactobacilli and infected by $E$. coli (ALE), however, no statistical difference was found ( $p \geq 0.05)$. In the groups only inoculated with alive L. rhamnosus (AL) or heat-killed (HK), there was a small mortality of larvae, differing statistically from the other groups $(p \leq 0.05)$.

In Fig. 2a, it was observed higher haemocyte count in the groups (ALS, ALE, HKS, HKE) previously inoculated with $L$. rhamnosus, alive or heat-killed, followed by inoculation with the pathogenic bacteria (S. aureus or E. coli), $(p<0.05)$ being statistically different from controls ( $\mathrm{Sa}$ and $\mathrm{Ec})$.

Figure $2 \mathrm{~b}$ shows the absorbance values found during the hemolymph melanization of larvae. It is possible to observe greater melanization in the groups stimulated with lactobacilli and infected with S. aureus or E. coli (ALS, ALE, HKS, HKE), differentiating statistically from the controls $(p<0.05)$ (Sa and Ec). The melanization in the group inoculated with $S$. aureus (ALS, HKS and Sa) was statically higher than those inoculated with $S$. aureus and heat-killed L. rhamnosus (Sa, HK and Control) $(p<0.05)$, which was similar to the group inoculated with $E$. coli (Ec) $(p>0.05)$. In the group stimulated only with alive $L$. rhamnosus (AL) the melanization was statistically higher than in the control group (Control) $(p>0.05)$. The group inoculated with heat-killed $L$. rhamnosus and infected with $S$. aureus (HKS) presented the highest optical density, with statistical difference when compared to all groups. This can also be observed regarding the production of nitric oxide (Fig. 2c). The other groups (AL, ALS, ALE, HK, HKE) were statistically similar to the control group $(p>0.05)$. 

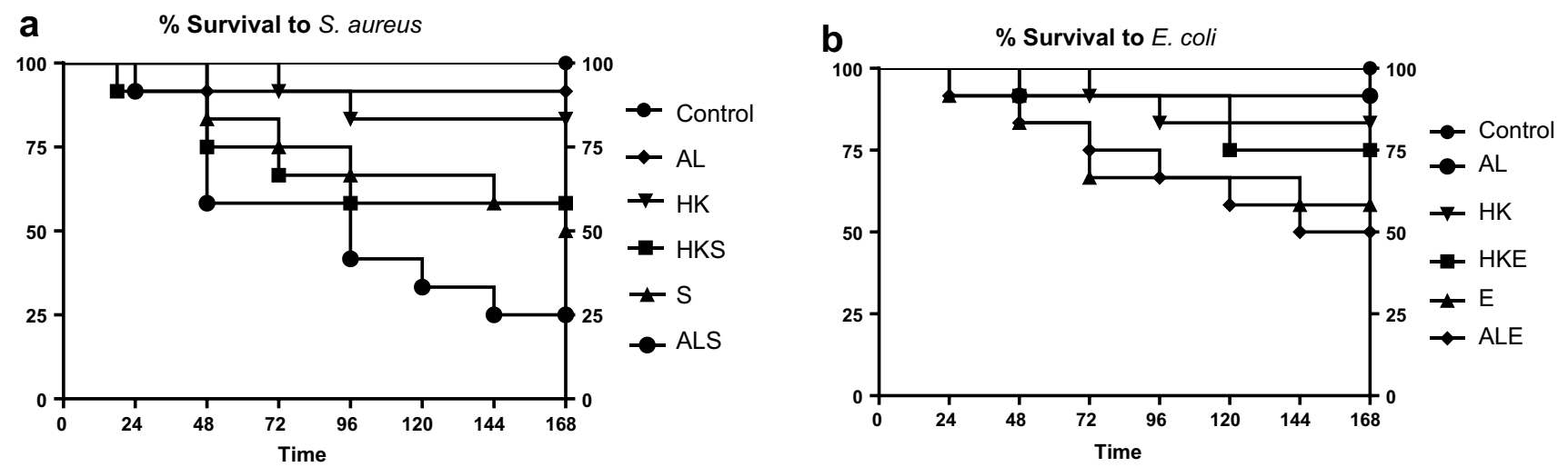

Fig. 1 a Survival curve of G. mellonella infected with S. aureus, with or without prior inoculation of alive or heat-killed L. rhamnosus; b Survival curve of G. mellonella infected with E. coli, with or without prior inoculation of alive or heat-killed L. rhamnosus
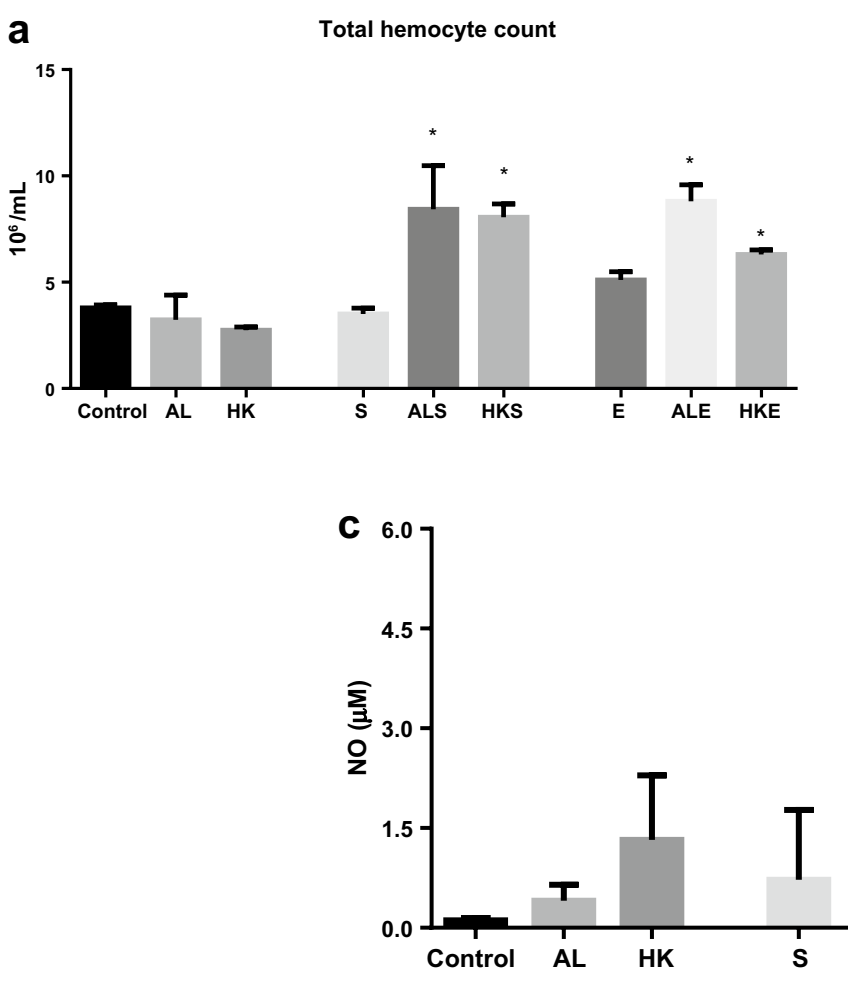

Fig. 2 a Mean and standard deviation of the total haemocyte count in G. mellonella hemolymph $24 \mathrm{~h}$ after inoculation of $S$. aureus and $E$. coli with or without prior inoculation of alive or heat-killed L. rhamnosus; $\mathbf{b}$ mean and standard deviation of hemolymph melanization in G. mellonella infected $24 \mathrm{~h}$ after the inoculation of $S$. aureus or $E$.

\section{Discussion}

Regarding the effects of alive or heat-killed L. rhamnosus on the response to $S$. aureus and E. coli, it can be observed that, although there is no statistical difference

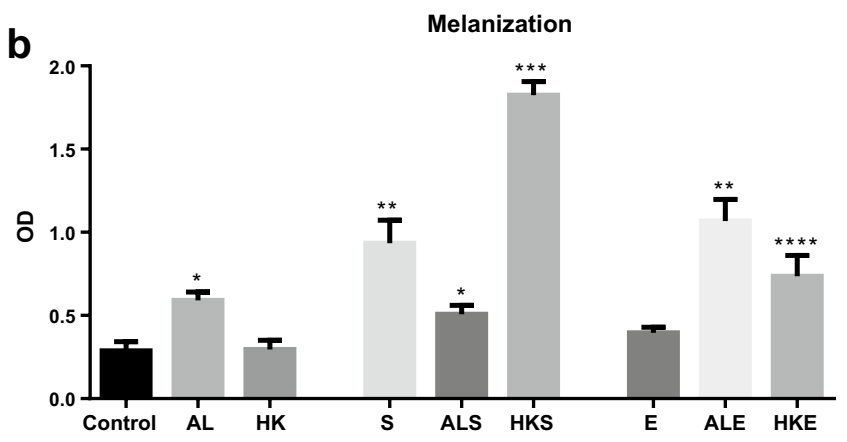

Nitric oxide

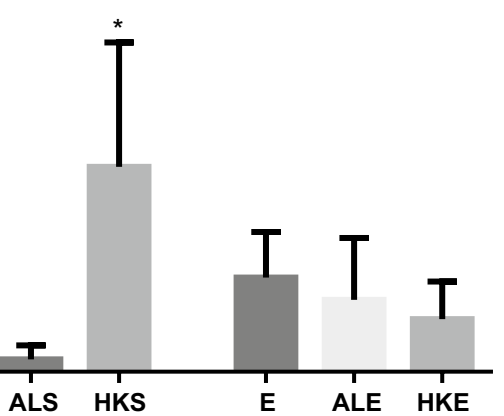

coli with or without prior inoculation of alive or heat-killed $L$. rhamnosus; c mean and standard deviation of nitric oxide (NO) concentration in G. mellonella hemolymph $24 \mathrm{~h}$ after inculation of $S$. aureus or $E$. coli with or without prior inoculation of alive or heat-killed $L$. rhamnosus

between the survival curves of the larvae, in the groups treated with heat-killed $L$. rhamnosus before pathogenic bacteria, the survival percentage of larvae increased. The same was not observed when the larvae were inoculated with alive L. rhamnosus, since it caused higher mortality when associated with pathogenic bacteria. The results also 
show that the mortality caused by inoculation of alive or heat-killed lactobacilli (12.5 and $8.39 \%$, respectively) was not significant, being similar to the control $(p \leq 0.05)$. In addition, heat-killed L. rhamnosus assisted in the response to $S$. aureus and E. coli. It was possible to observe an increase in the survival rate of the larvae, which was not observed when lactobacilli were alive, resulting in higher larvae mortality.

In a study using a probiotic strain of L. acidophilus alive and only its supernatant before infection with $C$. albicans in G. mellonella, the authors observed an increase in larvae survival. In addition, the number of $C$. albicans $(\mathrm{CFU} / \mathrm{mL})$ recuperated from larval hemolymph was lower in the group treated with L. acidophilus than in the control group, suggesting an increase in phagocytosis of this microorganism (Vilela et al. 2015). In this study, the authors used a period of $1 \mathrm{~h}$ period between inoculations of the microorganism and in our study this interval was $2.5 \mathrm{~h}$, since it was observed in previous in vitro studies, this is an optimal time for the induction of response in cell culture.

Insects do not have an adaptive response in the immune system, but they have an innate immune system that is based on recognition factors, which effectively and rapidly protects when there is an infection by microorganisms and parasites. This system includes cellular and humoral components that can be activated by invasion of foreign bodies, including pathogens (Hoffmann et al. 1995). In the cellular defense mechanism, haemocytes are able to recognize the threats and react in three different ways against pathogens: phagocytosis, nodule formation or encapsulation (Shaurub 2012). In our study, a higher density of haemocytes was observed in the hemolymph of the larvae previously inoculated with alive or heat-killed L. rhamnosus and later with $S$. aureus or E. coli, suggesting a higher response in these groups.

Fuchs et al. (2016) studied a prophylactic treatment with micafungin in G. mellonella infected with S. aureus (a pathogenic agent for which the antimicrobial does not demonstrate direct activity). The authors found that in the G. mellonella model, prophylactic treatment inhibited the action of pathogens and this was characterized by a 2.43 -fold increase of haemocytes density, compared to larvae inoculated only with PBS. They reported that this increase occurred $4 \mathrm{~h}$ after infection, and that the haemocytes density decreased over time and $24 \mathrm{~h}$ after antimicrobial injection, the increase was no more significant compared to the group inoculated with PBS. In our study, the total haemocytes count in the hemolymph was performed $24 \mathrm{~h}$ after inoculation of the microorganisms and this could explain why a decrease was observed in this count in the group inoculated with alive or heat-killed L. rhamnosus. In addition, Gibreel and Upton, in 2013, reported that a synthetic antimicrobial peptide, which has potent activity against Gram-positive microorganisms, protected G. mellonella larvae from S. aureus infection, but did not increase the total count haemocytes after $24 \mathrm{~h}$ of infection. They concluded that defense against infection may have been achieved by increasing the rate of phagocytosis. Rajendran et al. (2015) studied the effect of acetylcholine on the pathogenesis of C. albicans in G. mellonella and demonstrated that acetylcholine protected the larvae from infection and that this protection occurred by improving the function of haemocytes, demonstrating an increase in the total haemocyte count. The authors reported that this increase was observed $4 \mathrm{~h}$ after infection and that in $24 \mathrm{~h}$ there was a decrease. This leads us to believe that the hemocyte density in the groups inoculated with the bacteria could be higher if the counting was performed in less time after infection. Even so, our study was able to demonstrate that probiotic suspensions were able to induce greater response in the larvae. It also helps us to standardize the methodology of infection and study of this model, aiming to make it a source of reliable results in the study of bacteria with probiotic effects.

Galleria mellonella has a humoral defense mechanism with components that are involved in the recognition or are directly responsible for the immune response. These components are responsible for the synthesis and release of various antibacterial peptides. In addition, there are also other humoral components, including lectins (hemagglutinins) and a melanin-phenoloxidase (PO) system responsible for the coagulation of hemolymph (melanization) and death of microbial invaders, as well as parasites (Shaurub 2012; Zdybicka-Barabas et al. al. 2014). In our study, this response was evaluated by means of haemolymph melanization and the results demonstrated that this immune response was triggered after inoculation of alive or heat-killed lactobacilli, with a greater melanization in the lactobacilli-stimulated groups, suggesting that the inoculation of the probiotic bacteria triggered the sequence of responses, without causing the larvae death, modulating its response. In the groups stimulated only with $L$. rhamnosus, alive lactobacilli caused greater melanization and when the bacteria were heat-killed, the melanization was not statistically different $(p>0.05)$ from the control group. Wand et al. (2013), evaluated the response of $G$. mellonella to infection by several Klebsiella pneumoniae strains and reported that, in general, strains that caused a higher degree of mortality of larvae showed increased levels of melanization. Strains that stimulated low levels of melanization had an initial phenoloxidase (PO) activity, responsible for hemolymph coagulation, after $60 \mathrm{~min}$, but this activity did not remain after $4 \mathrm{~h}$, with the most virulent strains showing a significant increase in the levels of PO activity when compared to control group. These results corroborate with those found in our study, where it was shown that heat-killed L. rhamnosus only triggered the melanin-phenoloxidase (PO) system responsible for hemolymph coagulation (melanization) initially, but this 
response was not maintained, causing a low degree of mortality of larvae. In addition, in our study, higher hemolymph melanization was found in the groups inoculated with $S$. aureus, in the period of $24 \mathrm{~h}$, and this microorganism was shown to be the most pathogenic to the larvae, causing greater mortality and greater melanization of the larvae. These results demonstrate that heat-killed probiotic bacteria may induce a beneficial effect without causing damage to the host.

Regarding the production of nitric oxide, it could be observed that the only group in which there was higher nitric oxide production was the one infected treated with heatkilled $L$. rhamnosus and infected with $S$. aureus. It was also observed a decrease when the larvae were treated with $L$. rhamnosus and infected with $E$. coli, however, no statistical difference was found $(p \geq 0.05)$. The results for NO are in agreement with those found regarding melanization and haemocyte count. It could be observe that NO production was higher in the group treated with heat-killed probiotic, followed by the bacterium that showed to be more pathogenic. The groups in which heat-killed lactobacilli triggered hemolymph melanization, initially, had no maintenance of this response and when the larvae were infected with $S$. aureus the insect defense system recognized the threat and induced greater NO production, which is an important molecule in the immune response to microorganisms and parasites in insects. In addition, Semenova et al. (2014) reported that a quantitative analysis of NO production in insects is more complicated than in mammals due to a deceleration of metabolic activities and Whitten et al. (2007) observed that the NO-mediated immune response in insects is specific for each pathogen and that through inoculation of LPS, it was more remarkable in the body fat than in haemocytes.

Our study demonstrated that for this model of infection and under these experimental conditions, heat-killed $L$. rhamnosus was better at inducing a response and protection of larvae against $S$. aureus and $E$. coli. However, knowing that insects have very fast defense and metabolism systems, more studies are necessary ordering to evaluate whether adopting a shorter interval between inoculation of the microorganisms, the response of larvae to infection and the preventive effect of lactobacilli could be improved. In addition, it is known that the probiotic effect of bacteria is dose dependent, so other studies with different suspensions are necessary.

Through the results, we can observe the need for further studies of the effects of probiotic bacteria in G. mellonella, with different periods of infection and intervals between inoculations of probiotic and pathogenic bacteria. It was concluded that, for this infection model and under these experimental conditions, heat-killed L. rhamnosus ATCC 7469 promoted greater protection of larvae infected with $S$. aureus or E. coli.

\section{Compliance with ethical standards}

Funding This study was not funded.

Conflict of interest The authors declare no conflict of interest.

Ethical approval There is no need for ethical advice approval to use Galleria mellonella as an infection model. However, all institutional guidelines applicable to the care and use of the animals were followed.

\section{References}

Adams CA (2010) The probiotic paradox: live and dead cells are biological response modifiers. Nutr Res Rev 23(1):37-46. doi:10.1017/S0954422410000090

Dash G, Raman RP, Pani Prasad K, Makesh M, Pradeep MA, Sen S (2015) Evaluation of paraprobiotic applicability of Lactobacillus plantarum in improving the immune response and disease protection in giant freshwater prawn, Macrobrachium rosenbergii. Fish Shellfish Immunol 43(1):167 - 74. doi:10.1016/j.fsi.2014.12.007

Fuchs BB, Li Y, Li D, Johnston T, Hendricks G, Li G, Rajamuthiah R, Mylonakis E (2016) Micafungin elicits an immunomodulatory effect in Galleria mellonella and mice. Mycopathologia 181(12):17-25. doi:10.1007/s11046-015-9940-z

Gibreel TM, Upton M (2013 Oct) Synthetic epidermicin NI01 can protect Galleria mellonella larvae from infection with Staphylococcus aureus. J Antimicrob Chemother 68(10):2269-2273. doi:10.1093/jac/dkt195

Harb H, van Tol EA, Heine H, Braaksma M, Gross G, Overkamp K, Hennen M, Alrifai M, Conrad ML, Renz H, Garn H (2013) Neonatal supplementation of processed supernatant from Lactobacillus rhamnosus GG improves allergic airway inflammation in mice later in life. Clin Exp Allergy 43(3):353-364. doi:10.1111/ cea. 12047

Hoffmann JA (1995) Innate immunity of insects. Curr Opin Immunol $7(1): 4-10$

Jorjão AL, Oliveira FE, Leão MVP, Carvalho CAT, Jorge AOC, Oliveira LD (2015) Live and heat-killed Lactobacillus rhamnosus ATCC 7469 may induce modulatory cytokines profiles on macrophages RAW 264.7. Sci World J 2015:716749. doi: $10.1155 / 2015 / 716749$

Junqueira JC (2012) Galleria mellonella as a model host for human pathogens: recent studies and new perspectives. Virulence 3(6):474-476. doi:10.4161/viru.22493

Köhler G (2015) Probiotics research in Galleria mellonella. Virulence 6(1):3-5. doi:10.1080/21505594.2014.998967

Nakayama Y, Moriya T, Sakai F, Ikeda N, Shiozaki T, Hosoya T, Nakagawa H, Miyazaki T (2014) Oral administration of Lactobacillus gasseri SBT2055 is effective for preventing influenza in mice. Sci Rep 4:4638. doi:10.1038/srep04638

Rajendran R, Borghi E, Falleni M, Perdoni F, Tosi D, Lappin DF, O'Donnell L, Greetham D, Ramage G, Nile C (2015) Acetylcholine protects against Candida albicans infection by inhibiting biofilm formation and promoting hemocyte function in a $\mathrm{Gal}$ leria mellonella infection model. Eukaryot Cell 14(8):834-844. doi:10.1128/EC.00067-15

Ramarao N, Nielsen-Leroux C, Lereclus D (2012) The insect Galleria mellonella as a powerful infection model to investigate bacterial pathogenesis. J Vis Exp 70:e4392. doi:10.3791/4392

Semenova AD, Glazachev YI, Slepneva IA, Glupov VV (2014) Quantitative determination of nitric oxide production in haemocytes: nitrite reduction activity as a potential pathway of $\mathrm{NO}$ formation 
in haemolymph of Galleria mellonella larvae. Nitric Oxide 37:46-52. doi:10.1016/j.niox.2013.12.011

Shaurub EH (2012) Immunomodulation in insects post-treatment with abiotic agents: a review. Eur J Entomol 109(3):303-316

Taverniti V, Guglielmetti S (2011) The immunomodulatory properties of probiotic microorganisms beyond their viability (ghost probiotics: proposal of paraprobiotic concept). Genes Nutr 6(3):261 - 74 . doi:10.1007/s12263-011-0218-x

Vilela SF, Barbosa JO, Rossoni RD, Santos JD, Prata MC, Anbinder AL, Jorge AO, Junqueira JC (2015) Lactobacillus acidophilus ATCC 4356 inhibits biofilm formation by $C$. albicans and attenuates the experimental candidiasis in Galleria mellonella. Virulence 6(1):29-39. doi:10.4161/21505594.2014.981486

Wand ME, McCowen JW, Nugent PG, Sutton JM (2013) Use of the Galleria mellonella infection model to study the opportunistic pathogen Klebsiella pneumonia supports the presence of a multifaceted interaction with the host immune response. J Med Microbiol 62(Pt 12):1790-1798. doi:10.1099/jmm.0.063032-0

Whitten M, Sun F, Tew I, Schaub G, Soukou C, Nappi A, Ratcliffe N (2007) Differential modulation of Rhodnius prolixus nitric oxide activities following challenge with Trypanosoma rangeli, T. cruzi and bacterial cell wall components. Insect Biochem Mol Biol 37(5):440-452

WHO (2001) Health and nutritional properties of probiotics in food including powder milk with live lactic acid bacteria. Disponível em: http://www.who.int/foodsafety/publications/fs_management/ en/probiotics.pdf

Zdybicka-Barabas A, Mak P, Jakubowicz T, Cytryńska M (2014) Lysozyme and defense peptides as suppressors of phenoloxidase activity in Galleria mellonella. Arch Insect Biochem Physiol 87(1):1-12. doi:10.1002/arch.21175 